\title{
Room lighting in the absence of a defined visual task and the impact of mean room surface exitance
}

\author{
Peter Raynham \\ The UCL Institute for Environmental Design and Engineering \\ Central House, 14 Upper Woburn Place London WC1H 0NN \\ e: p.raynham@ucl.ac.uk \\ t: $\quad+44(0) 2031089029$
}

\begin{abstract}
With conditions for lighting design changing it is becoming important to consider what to do in rooms where there is no particular visual task. The current European standard for lighting in work places gives a number of requirements on room lighting the most onerous being the requirement for cylindrical illuminance. Mean room surface exitance (MRSE) has been put forward as a metric that is a good predictor of perceived adequacy of illumination. The paper examines the consequences of adopting a proposed MRSE target of 100 lumens per square meter in 2 sizes of room with a variety of surface reflectances and lit by an regular array of light sources with a range of distributions. The paper concludes that significant extra flux will be required to meet the new MRSE target and that light distributions with a significant upward component will also be needed.
\end{abstract}

\section{Introduction}

BS EN $12461-1^{1}$ provides the basis of much of the lighting design in the UK. However, often the designers only refer to the schedule of task illuminances given in section 5 of the standard and do not consider the other provisions of the document.

In many working environments the visual tasks have become easier over the past decade or so. This is particularly true of offices where self luminous screens are now the most 
common visual task; laser printed documents also being very common. Moreover the lighting for many buildings is specified and installed long before the user of the building has worked out where any visual tasks will take place. The current inappropriate practice for such situations is to provide light to a notional working plane at an illuminance that corresponds to the illuminance for the most demanding visual task that might be carried out in the space. The net result is that it is very often the case that too much light is given to visual tasks and very often the light for tasks is spread across an entire room, rather than just being provided where it is needed. Thus in an age where we are being encouraged to reduce energy consumption it makes much more sense to provide fixed lighting in a room to meet the minimum room lighting, and then provide localised task lighting for any particular tasks.

This gives rise the question: what are the minimum room lighting requirements?

Cuttle $^{2}$ has argued that perceived adequacy of illuminance is a key criteria for any well lit room. He has related that to the mean room surface exitance (MRSE) of room. Cuttle suggests that a MRSE value of 100 lux is required for an acceptably bright appearance for a room. BS EN $12464-1{ }^{1}$ has number of requirements that relate to the whole room. They are:

- Average wall illuminance to be 50 lux (75 lux in offices, education, health care and general areas of entrance, corridors, stairs, etc.)

- Average ceiling illuminance to be 30 lux (50 lux in offices, education, health care and general areas of entrance, corridors, stairs, etc.)

- Mean cylindrical illuminance at head height of 50 lux where visual communication is less important and 150 lux where it is important. 
- Modelling is defined by the ratio of cylindrical illuminance to horizontal illuminance at a given point it is recommended that this ratio should be in the range 0.3 to 0.6 if a regular array of light sources is being used.

BS EN 12464- $1^{1}$ also gives guidance on the range of room reflectances that may be used. Table 1 gives the recommended ranges of surface reflectance

\begin{tabular}{|c|c|}
\hline Surface & Reflectance Range \\
\hline Ceiling & 0.7 to 0.9 \\
\hline Walls & 0.3 to 0.8 \\
\hline Floor & 0.2 to 0.4 \\
\hline
\end{tabular}

Table 1 Recommended values of room surface reflectance from BS EN 12464-1

If MRSE is to be adopted in standards and codes it is necessary to understand how its use will impact on lighting design and in particular what changes it is likely to bring in the total fluxed used in a room, the distribution of light and room surface reflectances. Moreover, it is necessary to understand if a MRSE value of 100 lumens per square meter can be achieved without significantly increasing the total flux required to light a given room.

This paper explores the relationships between these parameters in 2 different room sizes; each with a range of surface reflectances and with a range of luminaire light distributions. 


\section{Methodology}

The objective in establishing this methodology is to develop a comparison method that will work in a number of generic situations, rather than select actual conditions found in a given room where standard conditions have been used.

\subsection{Room dimensions}

Two room sizes were studied; one with room index 2 and the other with room index 4. Some of the calculation methods used were based on those set out in EN 13032-2 3 which are defined for rooms where the length of the room is 1.6 times the width. The dimensions of the rooms are given in Table 2.

\begin{tabular}{|c|c|c|}
\hline Room Index (k) & 2 & 4 \\
\hline Length & 12.48 & 24.96 \\
\hline Width & 7.8 & 15.6 \\
\hline Height & 2.4 & 2.4 \\
\hline
\end{tabular}

Table 2 The length and width of the rooms

Note: in the calculation of room index the height between floor and ceiling is used as the concept of a working plane is not being employed.

\subsection{Surface reflectances}

In order to fully exploit the calculation methods set out in EN 13032-2 in this study, the values of surface reflectance used for the calculation of utilisation factors were used in this study. Table 3 gives the values of surface reflectance used in this study.

\begin{tabular}{|c|c|c|}
\hline Ceiling & Walls & Floor \\
\hline 0.8 & 0.7 & 0.2 \\
\hline 0.8 & 0.5 & 0.2 \\
\hline 0.8 & 0.3 & 0.2 \\
\hline
\end{tabular}




\begin{tabular}{|l|l|l|}
\hline 0.7 & 0.7 & 0.2 \\
\hline 0.7 & 0.5 & 0.2 \\
\hline 0.7 & 0.3 & 0.2 \\
\hline 0.5 & 0.7 & 0.2 \\
\hline 0.5 & 0.5 & 0.2 \\
\hline 0.5 & 0.3 & 0.2 \\
\hline
\end{tabular}

Table 3 The combinations of surface reflectance used

Note: the methods used in this study may be extended to any combination of room reflectance, but, it would be necessary to calculate new transfer factors for each new combination of reluctances using the method given by Raynham and Bean ${ }^{4}$

\subsection{Light sources}

For this study to have value it is necessary to examine the results achieved with a wide range of light sources. A system giving standardised light distributions was needed. The 10 light distributions of the British Zonal (BZ) system were adopted for this study. The BZ system was introduced by the Illuminating Engineering Society (IES), the precursor to the current Society of Light and Lighting, in the IES Code in 1961. The system describes the distribution from a light source as a function of the elevation $\gamma$ multiplied by a normalising constant so that the total flux emitted by each nominal light source is 1,000 lumens. By definition BZ distributions have only a downward light output however, in the study it was necessary to consider the impact of an upward component of light output. As it was taken that the luminaires were all ceiling mounted it was assumed that any upward flux went directly to the ceiling, so upward flux can simply be modelled by a reduction in downward flux distributed to the walls and floor and with an increase in the flux arriving at the ceiling.

The light sources were assumed to be mounted in a regular array on the ceiling of the room and spaced according to the spacing to height ratio. Table 4 gives the function 
and normalisation factor for each of the $10 \mathrm{BZ}$ distributions together with the maximum spacing to height ratio for each distribution.

\begin{tabular}{|c|c|c|c|}
\hline BZ Number & Function & $\begin{array}{c}\text { Normalisation } \\
\text { Factor }\end{array}$ & $\begin{array}{c}\text { SHR } \\
\text { Max }\end{array}$ \\
\hline 1 & $\cos ^{4} \gamma$ & 795.61 & 1.255 \\
\hline 2 & $\cos ^{3} \gamma$ & 636.52 & 1.405 \\
\hline 3 & $\cos ^{2} \gamma$ & 477.42 & 1.624 \\
\hline 4 & $\cos ^{1.5} \gamma$ & 397.86 & 1.779 \\
\hline 5 & $\cos \gamma$ & 318.36 & 1.989 \\
\hline 6 & $1+\cos \gamma$ & 106.11 & 2.381 \\
\hline 7 & $2+\cos \gamma$ & 63.66 & 2.512 \\
\hline 8 & 1 & 159.15 & 2.767 \\
\hline 9 & $1+\sin \gamma$ & 89.14 & 3.184 \\
\hline 10 & $\sin \gamma$ & 202.65 & 1.584 \\
\hline
\end{tabular}

Table 4 The function, normalisation factor and SHR for each of the BZ distributions.

Note: The spacing to height ratio given in Table 4 was calculated using the method set out in the National Annex to BS EN 13032-2 ${ }^{3}$ and Chapter 12 of the SLL Code for Lighting $^{5}$. Figure 1 is a polar plot of some of the BZ distributions.

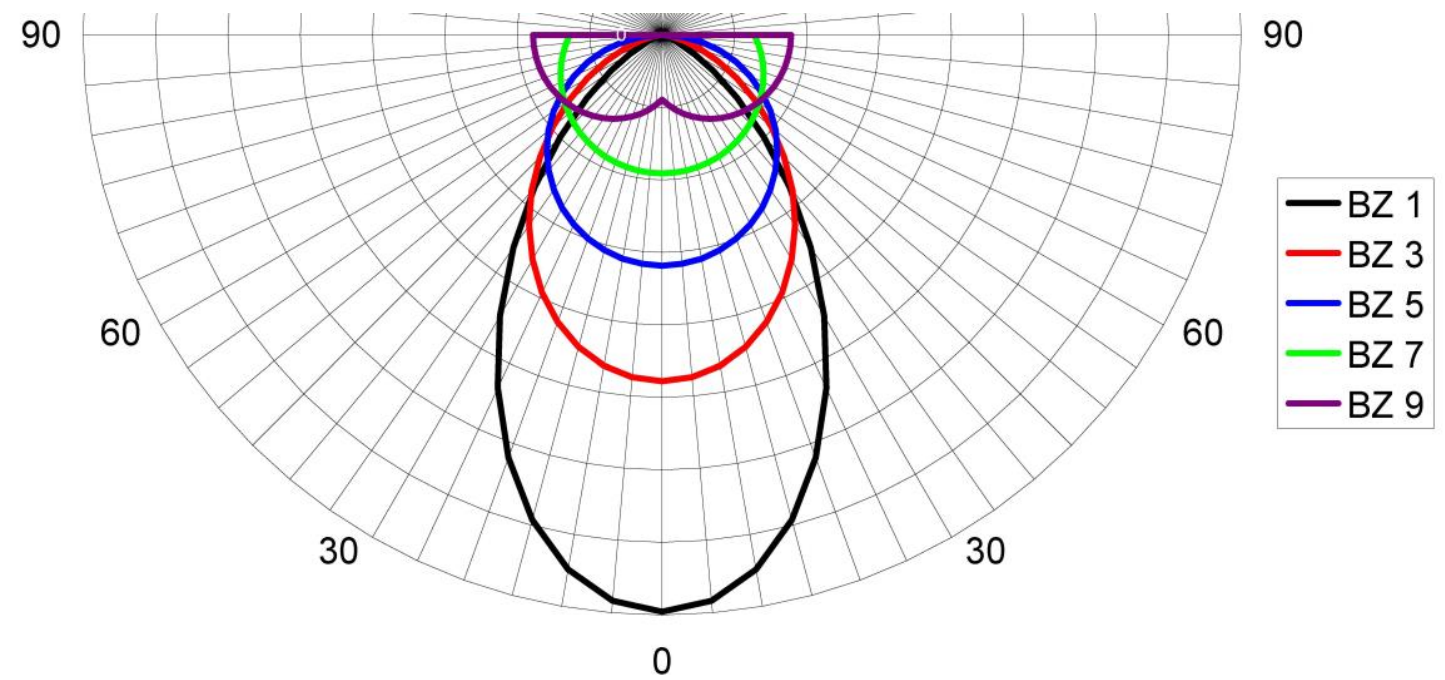

Figure 1 A polar plot of some of the $\mathrm{BZ}$ distributions 


\subsection{Calculation of the illuminance, luminance and exitance of the room surfaces}

Using the utilisation factor method set out in EN 13032-2 and using the extra sets of transfer factors from Raynham and Bean ${ }^{4}$, it is possible to calculate the utilization factors for each of the room surfaces. It is therefore possible to calculate the illuminance on each of the room surfaces by multiplying the nominal flux from the light sources by the utilisation factor and dividing by the area of the surface in question. The exitance of the surface may be calculated by multiplying the illuminance on the surface by reflectance of the surface. The surfaces are assumed to be have matt finishes and that the surface luminance is the same in all directions, hence the luminance of the surface may be calculated by dividing the exitance by pi.

\subsection{Calculation of cylindrical illuminance and modelling}

In EN 12464-1 ${ }^{1}$ it does not stipulate exactly where the cylindrical illuminance should be calculated. That said, it would be reasonable practice to evaluate cylindrical illuminance at each point on the CEN grid parallel to the floor of the room at a height of $1.2 \mathrm{~m}$ which corresponds to the face height of a seated person. The CEN grid is defined by Equation 1 which is from section 4.1 .4 of EN 12464- $1^{1}$

$$
p=0.2 \times 5^{\log _{10}(d)}
$$

\section{Equation 1}

In Equation $1 p$ is the maximum distance between grid points and $d$ is the longer dimension of the room. A border of $0.5 \mathrm{~m}$ around the edge of the room is excluded from the calculation. Table 5 gives the number and disposition of points used: 


\begin{tabular}{|c|c|c|}
\hline Room Index & 2 & 4 \\
\hline Number of points in length & 11 & 14 \\
\hline Spacing in length & 1.044 & 1.711 \\
\hline First point in length & 1.022 & 1.356 \\
\hline Number of points in width & 7 & 8 \\
\hline Spacing in width & 0.971 & 1.825 \\
\hline First Point in width & 0.986 & 1.413 \\
\hline
\end{tabular}

Table 5 The number, spacing and distance for first grid point from wall for the calculation points

The calculation of cylindrical illuminance uses the formula given in Equation 2

$$
E_{c y l}=\frac{I \sin (\theta)}{D^{2} \pi}
$$

\section{Equation 2}

Where $E_{c y l}$ is the cylindrical illuminance at a point that is due to intensity $I$ from a source that is distance $D$ away from the point being illuminated and that subtends an angle $\theta$ to the vertical at the point. The process of calculating the direct cylindrical illuminance from the light sources is relatively easy. The indirect part of the cylindrical illuminance calculation is more complex. It is necessary to divide the room surfaces up into small sections so that it becomes possible to treat each element of the surface as a point source. The calculation then proceeds by evaluating the projected area of the surface towards the point where the illuminance is being evaluated, then the intensity is given by the surface luminance multiplied by the projected area. The calculations can be simplified as they only have to be done once for each room surface with a nominal surface luminance then the final value can be obtained by multiplying by the ratio of actual to nominal surface luminance. The situation is also simplified as the point at which the cylindrical illuminance is being calculated is equidistant from floor and ceiling, so the relative contribution from the floor is the same as that of the ceiling. 


\section{Results}

\subsection{Utilization factors}

Table 6, Table 7 and Table 8 give the utilization factors for the floor, walls and ceiling in the space with room index 2.

\begin{tabular}{|c|c|c|c|c|c|c|c|c|c|}
\hline \multicolumn{10}{|c|}{ Reflectances } \\
\hline $\mathrm{C}$ & 0.8 & 0.8 & 0.8 & 0.7 & 0.7 & 0.7 & 0.5 & 0.5 & 0.5 \\
$\mathrm{~W}$ & 0.7 & 0.5 & 0.3 & 0.7 & 0.5 & 0.3 & 0.7 & 0.5 & 0.3 \\
$\mathrm{~F}$ & 0.2 & 0.2 & 0.2 & 0.2 & 0.2 & 0.2 & 0.2 & 0.2 & 0.2 \\
\hline $\mathrm{BZ}$ & \multicolumn{10}{|c|}{} \\
\hline 1 & 1.068 & 1.017 & 0.976 & 1.048 & 1.002 & 0.965 & 1.011 & 0.974 & 0.943 \\
\hline 2 & 1.048 & 0.989 & 0.943 & 1.027 & 0.974 & 0.931 & 0.988 & 0.945 & 0.909 \\
\hline 3 & 1.030 & 0.965 & 0.913 & 1.008 & 0.949 & 0.902 & 0.967 & 0.919 & 0.880 \\
\hline 4 & 1.011 & 0.940 & 0.883 & 0.989 & 0.924 & 0.871 & 0.947 & 0.893 & 0.849 \\
\hline 5 & 0.978 & 0.894 & 0.828 & 0.953 & 0.878 & 0.816 & 0.909 & 0.846 & 0.794 \\
\hline 6 & 0.916 & 0.811 & 0.727 & 0.889 & 0.793 & 0.715 & 0.840 & 0.760 & 0.693 \\
\hline 7 & 0.900 & 0.789 & 0.701 & 0.873 & 0.771 & 0.689 & 0.822 & 0.737 & 0.667 \\
\hline 8 & 0.876 & 0.757 & 0.661 & 0.847 & 0.738 & 0.649 & 0.794 & 0.703 & 0.627 \\
\hline 9 & 0.853 & 0.725 & 0.623 & 0.823 & 0.706 & 0.611 & 0.768 & 0.670 & 0.589 \\
\hline 10 & 0.808 & 0.665 & 0.550 & 0.777 & 0.645 & 0.538 & 0.718 & 0.608 & 0.516 \\
\hline
\end{tabular}

Table 6 Utilization factors to the floor for room index $=2$

\begin{tabular}{|c|c|c|c|c|c|c|c|c|c|}
\hline \multicolumn{10}{|c|}{ Reflectances } \\
\hline C & 0.8 & 0.8 & 0.8 & 0.7 & 0.7 & 0.7 & 0.5 & 0.5 & 0.5 \\
W & 0.7 & 0.5 & 0.3 & 0.7 & 0.5 & 0.3 & 0.7 & 0.5 & 0.3 \\
F & 0.2 & 0.2 & 0.2 & 0.2 & 0.2 & 0.2 & 0.2 & 0.2 & 0.2 \\
\hline BZ & \multicolumn{10}{|c|}{} \\
\hline 1 & 0.337 & 0.299 & 0.269 & 0.323 & 0.289 & 0.261 & 0.298 & 0.270 & 0.247 \\
\hline 2 & 0.385 & 0.342 & 0.307 & 0.370 & 0.331 & 0.300 & 0.344 & 0.312 & 0.285 \\
\hline 3 & 0.427 & 0.379 & 0.341 & 0.412 & 0.369 & 0.334 & 0.385 & 0.349 & 0.319 \\
\hline 4 & 0.470 & 0.418 & 0.376 & 0.455 & 0.407 & 0.368 & 0.426 & 0.386 & 0.353 \\
\hline 5 & 0.549 & 0.488 & 0.439 & 0.533 & 0.477 & 0.431 & 0.502 & 0.455 & 0.416 \\
\hline 6 & 0.694 & 0.616 & 0.554 & 0.675 & 0.604 & 0.546 & 0.641 & 0.581 & 0.532 \\
\hline 7 & 0.731 & 0.649 & 0.584 & 0.712 & 0.637 & 0.576 & 0.678 & 0.614 & 0.562 \\
\hline 8 & 0.788 & 0.700 & 0.629 & 0.768 & 0.687 & 0.622 & 0.732 & 0.664 & 0.607 \\
\hline 9 & 0.843 & 0.748 & 0.673 & 0.822 & 0.736 & 0.665 & 0.785 & 0.712 & 0.651 \\
\hline 10 & 0.947 & 0.841 & 0.756 & 0.925 & 0.828 & 0.749 & 0.886 & 0.803 & 0.734 \\
\hline
\end{tabular}

Table 7 Utilization factors to the walls for room index $=2$ 


\begin{tabular}{|c|c|c|c|c|c|c|c|c|c|}
\hline \multicolumn{10}{|c|}{ Reflectances } \\
\hline C & 0.8 & 0.8 & 0.8 & 0.7 & 0.7 & 0.7 & 0.5 & 0.5 & 0.5 \\
\hline W & 0.7 & 0.5 & 0.3 & 0.7 & 0.5 & 0.3 & 0.7 & 0.5 & 0.3 \\
\hline $\mathrm{F}$ & 0.2 & 0.2 & 0.2 & 0.2 & 0.2 & 0.2 & 0.2 & 0.2 & 0.2 \\
\hline \multicolumn{10}{|l|}{ BZ } \\
\hline 1 & 0.222 & 0.184 & 0.153 & 0.216 & 0.180 & 0.151 & 0.204 & 0.173 & 0.146 \\
\hline 2 & 0.232 & 0.188 & 0.153 & 0.225 & 0.184 & 0.151 & 0.214 & 0.177 & 0.146 \\
\hline 3 & 0.240 & 0.192 & 0.153 & 0.234 & 0.188 & 0.151 & 0.221 & 0.180 & 0.146 \\
\hline 4 & 0.249 & 0.196 & 0.153 & 0.242 & 0.192 & 0.151 & 0.230 & 0.184 & 0.146 \\
\hline 5 & 0.265 & 0.203 & 0.153 & 0.258 & 0.199 & 0.151 & 0.244 & 0.191 & 0.146 \\
\hline 6 & 0.295 & 0.216 & 0.153 & 0.287 & 0.212 & 0.151 & 0.272 & 0.203 & 0.146 \\
\hline 7 & 0.302 & 0.219 & 0.153 & 0.294 & 0.215 & 0.151 & 0.279 & 0.206 & 0.146 \\
\hline 8 & 0.314 & 0.225 & 0.153 & 0.305 & 0.220 & 0.151 & 0.289 & 0.211 & 0.146 \\
\hline 9 & 0.325 & 0.229 & 0.153 & 0.316 & 0.225 & 0.151 & 0.300 & 0.216 & 0.146 \\
\hline 10 & 0.346 & 0.239 & 0.153 & 0.337 & 0.234 & 0.151 & 0.319 & 0.225 & 0.146 \\
\hline
\end{tabular}

Table 9, Table 10 and Table 11 give the utilization factors for the floor, walls and ceiling in the space with room index 4.

\begin{tabular}{|c|c|c|c|c|c|c|c|c|c|}
\hline \multicolumn{10}{|c|}{ Reflectances } \\
\hline C & 0.8 & 0.8 & 0.8 & 0.7 & 0.7 & 0.7 & 0.5 & 0.5 & 0.5 \\
W & 0.7 & 0.5 & 0.3 & 0.7 & 0.5 & 0.3 & 0.7 & 0.5 & 0.3 \\
F & 0.2 & 0.2 & 0.2 & 0.2 & 0.2 & 0.2 & 0.2 & 0.2 & 0.2 \\
\hline BZ & \multicolumn{10}{|c|}{} \\
\hline 1 & 1.129 & 1.099 & 1.072 & 1.106 & 1.079 & 1.055 & 1.062 & 1.041 & 1.022 \\
\hline 2 & 1.118 & 1.084 & 1.054 & 1.094 & 1.063 & 1.036 & 1.050 & 1.025 & 1.003 \\
\hline 3 & 1.107 & 1.067 & 1.032 & 1.082 & 1.046 & 1.015 & 1.036 & 1.008 & 0.982 \\
\hline 4 & 1.097 & 1.053 & 1.015 & 1.072 & 1.033 & 0.998 & 1.025 & 0.993 & 0.965 \\
\hline 5 & 1.076 & 1.024 & 0.979 & 1.050 & 1.003 & 0.961 & 1.001 & 0.963 & 0.928 \\
\hline 6 & 1.022 & 0.948 & 0.883 & 0.993 & 0.926 & 0.866 & 0.939 & 0.883 & 0.833 \\
\hline 7 & 1.010 & 0.931 & 0.862 & 0.980 & 0.908 & 0.844 & 0.925 & 0.865 & 0.812 \\
\hline 8 & 0.992 & 0.905 & 0.829 & 0.961 & 0.882 & 0.812 & 0.904 & 0.838 & 0.779 \\
\hline 9 & 0.976 & 0.882 & 0.800 & 0.944 & 0.859 & 0.783 & 0.885 & 0.814 & 0.751 \\
\hline 10 & 0.949 & 0.844 & 0.752 & 0.915 & 0.820 & 0.735 & 0.854 & 0.774 & 0.703 \\
\hline
\end{tabular}

Table 9 Utilization factors to the floor for room index $=4$ 


\begin{tabular}{|c|l|l|l|l|l|l|l|l|l|}
\hline \multicolumn{10}{|c|}{ Reflectances } \\
\hline $\mathrm{C}$ & 0.8 & 0.8 & 0.8 & 0.7 & 0.7 & 0.7 & 0.5 & 0.5 & 0.5 \\
$\mathrm{~W}$ & 0.7 & 0.5 & 0.3 & 0.7 & 0.5 & 0.3 & 0.7 & 0.5 & 0.3 \\
$\mathrm{~F}$ & 0.2 & 0.2 & 0.2 & 0.2 & 0.2 & 0.2 & 0.2 & 0.2 & 0.2 \\
\hline
\end{tabular}

\begin{tabular}{|c|c|c|c|c|c|c|c|c|c|}
\hline \multicolumn{10}{|c|}{ BZ } \\
\hline 1 & 0.220 & 0.206 & 0.193 & 0.196 & 0.184 & 0.174 & 0.152 & 0.144 & 0.137 \\
\hline 2 & 0.232 & 0.217 & 0.204 & 0.208 & 0.196 & 0.184 & 0.164 & 0.155 & 0.148 \\
\hline 3 & 0.246 & 0.230 & 0.216 & 0.222 & 0.208 & 0.196 & 0.178 & 0.168 & 0.160 \\
\hline 4 & 0.257 & 0.240 & 0.226 & 0.233 & 0.219 & 0.206 & 0.189 & 0.179 & 0.169 \\
\hline 5 & 0.281 & 0.263 & 0.247 & 0.257 & 0.241 & 0.227 & 0.212 & 0.201 & 0.191 \\
\hline 6 & 0.344 & 0.321 & 0.301 & 0.319 & 0.299 & 0.282 & 0.273 & 0.259 & 0.245 \\
\hline 7 & 0.358 & 0.334 & 0.314 & 0.333 & 0.312 & 0.294 & 0.287 & 0.272 & 0.258 \\
\hline 8 & 0.379 & 0.354 & 0.332 & 0.354 & 0.332 & 0.313 & 0.308 & 0.291 & 0.276 \\
\hline 9 & 0.398 & 0.372 & 0.349 & 0.373 & 0.350 & 0.329 & 0.326 & 0.309 & 0.293 \\
\hline 10 & 0.429 & 0.401 & 0.376 & 0.404 & 0.379 & 0.357 & 0.357 & 0.338 & 0.320 \\
\hline
\end{tabular}

Table 10 Utilization factors to the walls for room index $=4$

\begin{tabular}{|c|c|c|c|c|c|c|c|c|c|}
\hline \multicolumn{10}{|c|}{ Reflectances } \\
\hline $\mathrm{C}$ & 0.8 & 0.8 & 0.8 & 0.7 & 0.7 & 0.7 & 0.5 & 0.5 & 0.5 \\
$\mathrm{~W}$ & 0.7 & 0.5 & 0.3 & 0.7 & 0.5 & 0.3 & 0.7 & 0.5 & 0.3 \\
$\mathrm{~F}$ & 0.2 & 0.2 & 0.2 & 0.2 & 0.2 & 0.2 & 0.2 & 0.2 & 0.2 \\
\hline $\mathrm{BZ}$ & \multicolumn{10}{|c|}{} \\
\hline 1 & 0.228 & 0.207 & 0.188 & 0.222 & 0.202 & 0.185 & 0.212 & 0.194 & 0.178 \\
\hline 2 & 0.234 & 0.209 & 0.188 & 0.228 & 0.205 & 0.185 & 0.217 & 0.196 & 0.178 \\
\hline 3 & 0.240 & 0.212 & 0.188 & 0.234 & 0.208 & 0.184 & 0.222 & 0.199 & 0.178 \\
\hline 4 & 0.245 & 0.214 & 0.187 & 0.239 & 0.210 & 0.184 & 0.227 & 0.201 & 0.177 \\
\hline 5 & 0.256 & 0.219 & 0.187 & 0.250 & 0.215 & 0.184 & 0.237 & 0.206 & 0.177 \\
\hline 6 & 0.284 & 0.232 & 0.186 & 0.277 & 0.227 & 0.182 & 0.263 & 0.217 & 0.176 \\
\hline 7 & 0.291 & 0.235 & 0.185 & 0.283 & 0.230 & 0.182 & 0.269 & 0.220 & 0.176 \\
\hline 8 & 0.300 & 0.239 & 0.185 & 0.293 & 0.234 & 0.182 & 0.278 & 0.224 & 0.175 \\
\hline 9 & 0.309 & 0.243 & 0.185 & 0.301 & 0.237 & 0.181 & 0.286 & 0.227 & 0.175 \\
\hline 10 & 0.323 & 0.249 & 0.184 & 0.315 & 0.244 & 0.181 & 0.299 & 0.233 & 0.174 \\
\hline
\end{tabular}

Table 11 Utilization factors to the ceiling for room index $=4$

For any given total flux leaving the light sources using tables 5 to 10 , it is possible to calculate the illuminance on each of the room surfaces and the exitance of each of the surfaces, and therefore the mean room surface exitance (MRSE). Table 12 and Table 13 give the MRSE values per 1000 installed lumens for room index 2 and 4 respectively. 


\begin{tabular}{|c|c|c|c|c|c|c|c|c|c|}
\hline \multicolumn{10}{|c|}{ Reflectances } \\
\hline$C$ & 0.8 & 0.8 & 0.8 & 0.7 & 0.7 & 0.7 & 0.5 & 0.5 & 0.5 \\
\hline W & 0.7 & 0.5 & 0.3 & 0.7 & 0.5 & 0.3 & 0.7 & 0.5 & 0.3 \\
\hline $\mathrm{F}$ & 0.2 & 0.2 & 0.2 & 0.2 & 0.2 & 0.2 & 0.2 & 0.2 & 0.2 \\
\hline \multicolumn{10}{|l|}{ BZ } \\
\hline 1 & 2.146 & 1.712 & 1.364 & 2.009 & 1.612 & 1.291 & 1.755 & 1.424 & 1.149 \\
\hline 2 & 2.275 & 1.778 & 1.381 & 2.131 & 1.676 & 1.307 & 1.866 & 1.483 & 1.166 \\
\hline 3 & 2.387 & 1.836 & 1.395 & 2.238 & 1.731 & 1.321 & 1.964 & 1.535 & 1.180 \\
\hline 4 & 2.502 & 1.895 & 1.410 & 2.348 & 1.789 & 1.336 & 2.063 & 1.589 & 1.195 \\
\hline 5 & 2.713 & 2.004 & 1.437 & 2.548 & 1.893 & 1.363 & 2.245 & 1.686 & 1.222 \\
\hline 6 & 3.097 & 2.202 & 1.486 & 2.914 & 2.084 & 1.413 & 2.578 & 1.863 & 1.272 \\
\hline 7 & 3.198 & 2.253 & 1.499 & 3.010 & 2.134 & 1.426 & 2.665 & 1.910 & 1.284 \\
\hline 8 & 3.349 & 2.331 & 1.519 & 3.154 & 2.209 & 1.445 & 2.795 & 1.980 & 1.304 \\
\hline 9 & 3.494 & 2.406 & 1.537 & 3.293 & 2.282 & 1.464 & 2.921 & 2.047 & 1.322 \\
\hline 10 & 3.773 & 2.550 & 1.573 & 3.558 & 2.420 & 1.499 & 3.162 & 2.176 & 1.358 \\
\hline
\end{tabular}

Table 12 MRSE values (lux) per 1,000 installed lumens for room index =2

\begin{tabular}{|c|c|c|c|c|c|c|c|c|c|}
\hline \multicolumn{10}{|c|}{ Reflectances } \\
\hline C & 0.8 & 0.8 & 0.8 & 0.7 & 0.7 & 0.7 & 0.5 & 0.5 & 0.5 \\
W & 0.7 & 0.5 & 0.3 & 0.7 & 0.5 & 0.3 & 0.7 & 0.5 & 0.3 \\
F & 0.2 & 0.2 & 0.2 & 0.2 & 0.2 & 0.2 & 0.2 & 0.2 & 0.2 \\
\hline BZ & \multicolumn{10}{|c|}{} \\
\hline 1 & 0.542 & 0.478 & 0.421 & 0.504 & 0.446 & 0.394 & 0.434 & 0.386 & 0.343 \\
\hline 2 & 0.562 & 0.488 & 0.424 & 0.523 & 0.456 & 0.397 & 0.451 & 0.395 & 0.345 \\
\hline 3 & 0.585 & 0.500 & 0.426 & 0.545 & 0.468 & 0.399 & 0.471 & 0.406 & 0.348 \\
\hline 4 & 0.603 & 0.510 & 0.429 & 0.562 & 0.477 & 0.402 & 0.487 & 0.415 & 0.351 \\
\hline 5 & 0.642 & 0.531 & 0.434 & 0.600 & 0.497 & 0.407 & 0.521 & 0.434 & 0.356 \\
\hline 6 & 0.744 & 0.586 & 0.447 & 0.697 & 0.550 & 0.420 & 0.609 & 0.483 & 0.370 \\
\hline 7 & 0.767 & 0.598 & 0.450 & 0.719 & 0.562 & 0.423 & 0.629 & 0.494 & 0.373 \\
\hline 8 & 0.802 & 0.617 & 0.454 & 0.752 & 0.580 & 0.428 & 0.659 & 0.511 & 0.377 \\
\hline 9 & 0.832 & 0.633 & 0.458 & 0.781 & 0.596 & 0.432 & 0.686 & 0.525 & 0.381 \\
\hline 10 & 0.884 & 0.660 & 0.465 & 0.830 & 0.622 & 0.438 & 0.730 & 0.550 & 0.388 \\
\hline
\end{tabular}

Table 13 MRSE values (lux) per 1,000 installed lumens for room index =4 


\subsection{Direct Cylindrical Illuminance}

The cylindrical illuminance at each grid point, $1.2 \mathrm{~m}$ above the floor, was calculated at each grid point for each of the light distributions and the average values for the results is given in Table 14

\begin{tabular}{|c|c|c|}
\hline \multirow{2}{*}{ Distribution } & \multicolumn{2}{|c|}{ Room Index } \\
\cline { 2 - 3 } & 2 & 4 \\
\hline BZ1 & 11.73 & 3.06 \\
\hline BZ2 & 11.23 & 3.11 \\
\hline BZ3 & 10.76 & 2.60 \\
\hline BZ4 & 11.43 & 2.45 \\
\hline BZ5 & 10.73 & 2.44 \\
\hline BZ6 & 8.96 & 2.21 \\
\hline BZ7 & 8.60 & 2.18 \\
\hline BZ8 & 8.34 & 2.20 \\
\hline BZ9 & 7.69 & 2.18 \\
\hline BZ10 & 6.61 & 2.02 \\
\hline
\end{tabular}

Table 14 Average cylindrical illuminance per 1,000 installed lumens for each of the BZ distributions

\subsection{Indirect cylindrical illuminance}

Indirect cylindrical illuminance at the grid points due to light leaving the ceiling, floor and the walls was calculated for the condition where the surface radiating light had a uniform luminance of $1 \mathrm{~cd} \mathrm{~m}^{-2}$. The average indirect cylindrical illuminance for the two sizes of room for the walls and the floor/ceiling are given in Table 15

\begin{tabular}{|l|c|c|}
\hline Surface & $\mathrm{RI}=2$ & $\mathrm{RI}=4$ \\
\hline Walls & 1.2510 & 0.7954 \\
\hline Floor or Ceiling & 0.9448 & 1.1725 \\
\hline
\end{tabular}

Table 15 Average indirect cylindrical illuminance where the luminance of the emitting surface is $1 \mathrm{~cd} \mathrm{~m}^{-2}$ 


\subsection{Overall cylindrical illuminance}

Based on the calculations for direct cylindrical illuminance and indirect cylindrical illuminance, coupled with the utilization factors on the room surfaces the overall cylindrical illuminance was calculated based on 1,000 installed lumens in each room. Table 16 and Table 17 give the value of overall cylindrical illuminance for rooms with room index 2 and 4 respectively.

\begin{tabular}{|c|c|c|c|c|c|c|c|c|c|}
\hline \multicolumn{10}{|c|}{ Reflectances } \\
\hline C & 0.8 & 0.8 & 0.8 & 0.7 & 0.7 & 0.7 & 0.5 & 0.5 & 0.5 \\
W & 0.7 & 0.5 & 0.3 & 0.7 & 0.5 & 0.3 & 0.7 & 0.5 & 0.3 \\
F & 0.2 & 0.2 & 0.2 & 0.2 & 0.2 & 0.2 & 0.2 & 0.2 & 0.2 \\
\hline BZ & \multicolumn{10}{|c|}{} \\
\hline 1 & 4.411 & 3.932 & 3.549 & 4.277 & 3.837 & 3.480 & 4.031 & 3.659 & 3.349 \\
\hline 2 & 4.962 & 4.413 & 3.976 & 4.819 & 4.315 & 3.908 & 4.564 & 4.133 & 3.775 \\
\hline 3 & 5.376 & 4.770 & 4.285 & 5.233 & 4.672 & 4.218 & 4.965 & 4.483 & 4.084 \\
\hline 4 & 5.618 & 4.952 & 4.417 & 5.470 & 4.851 & 4.348 & 5.192 & 4.658 & 4.215 \\
\hline 5 & 6.118 & 5.338 & 4.715 & 5.959 & 5.235 & 4.646 & 5.662 & 5.034 & 4.513 \\
\hline 6 & 6.686 & 5.699 & 4.911 & 6.507 & 5.588 & 4.842 & 6.178 & 5.375 & 4.710 \\
\hline 7 & 6.823 & 5.784 & 4.955 & 6.642 & 5.671 & 4.886 & 6.308 & 5.457 & 4.754 \\
\hline 8 & 7.171 & 6.053 & 5.156 & 6.980 & 5.934 & 5.088 & 6.631 & 5.716 & 4.955 \\
\hline 9 & 7.465 & 6.264 & 5.309 & 7.266 & 6.148 & 5.240 & 6.906 & 5.924 & 5.108 \\
\hline 10 & 7.683 & 6.339 & 5.262 & 7.475 & 6.215 & 5.195 & 7.090 & 5.982 & 5.062 \\
\hline
\end{tabular}

Table 16 Average overall cylindrical illuminance per 1,000 installed lumens for room index =2 


\begin{tabular}{|c|c|c|c|c|c|c|c|c|c|}
\hline \multicolumn{10}{|c|}{ Reflectances } \\
\hline C & 0.8 & 0.8 & 0.8 & 0.7 & 0.7 & 0.7 & 0.5 & 0.5 & 0.5 \\
\hline W & 0.7 & 0.5 & 0.3 & 0.7 & 0.5 & 0.3 & 0.7 & 0.5 & 0.3 \\
\hline $\mathrm{F}$ & 0.2 & 0.2 & 0.2 & 0.2 & 0.2 & 0.2 & 0.2 & 0.2 & 0.2 \\
\hline \multicolumn{10}{|l|}{$\mathrm{BZ}$} \\
\hline 1 & 1.106 & 1.033 & 0.968 & 1.069 & 1.002 & 0.943 & 1.001 & 0.944 & 0.893 \\
\hline 2 & 1.206 & 1.121 & 1.048 & 1.168 & 1.090 & 1.022 & 1.097 & 1.031 & 0.973 \\
\hline 3 & 1.403 & 1.307 & 1.223 & 1.364 & 1.276 & 1.197 & 1.292 & 1.217 & 1.148 \\
\hline 4 & 1.540 & 1.434 & 1.341 & 1.500 & 1.402 & 1.315 & 1.426 & 1.342 & 1.266 \\
\hline 5 & 1.709 & 1.582 & 1.471 & 1.668 & 1.550 & 1.446 & 1.590 & 1.488 & 1.397 \\
\hline 6 & 2.065 & 1.885 & 1.727 & 2.020 & 1.851 & 1.701 & 1.934 & 1.785 & 1.652 \\
\hline 7 & 2.134 & 1.940 & 1.771 & 2.086 & 1.906 & 1.746 & 1.998 & 1.840 & 1.697 \\
\hline 8 & 2.237 & 2.026 & 1.840 & 2.189 & 1.991 & 1.815 & 2.098 & 1.923 & 1.767 \\
\hline 9 & 2.347 & 2.120 & 1.920 & 2.297 & 2.084 & 1.895 & 2.204 & 2.016 & 1.847 \\
\hline 10 & 2.438 & 2.183 & 1.960 & 2.386 & 2.146 & 1.935 & 2.288 & 2.076 & 1.887 \\
\hline
\end{tabular}

Table 17 Average overall cylindrical illuminance per 1,000 installed lumens for room index =4

\section{Analysis and Discussion}

The results from the calculations give a basis for the comparison of the impacts of different possible metrics that may be used for the specification. Consider a room that is to be used as an office, from EN 12464- $1^{1}$ it would be expected to have cylindrical illuminance of 150 lux and an average illuminance on the walls and ceiling of 75 and 50 lux respectively. It also could be argued that it should have a mean room surface exitance of 100 lux. So it makes sense to think about how much luminaire flux is required in each room in order to achieve these objectives. Table 18 and Table 19 give the required total flux in the room to achieve the cylindrical illuminance target. 


\begin{tabular}{|c|c|c|c|c|c|c|c|c|c|}
\hline \multicolumn{10}{|c|}{ Reflectances } \\
\hline $\mathrm{C}$ & 0.8 & 0.8 & 0.8 & 0.7 & 0.7 & 0.7 & 0.5 & 0.5 & 0.5 \\
$\mathrm{~W}$ & 0.7 & 0.5 & 0.3 & 0.7 & 0.5 & 0.3 & 0.7 & 0.5 & 0.3 \\
$\mathrm{~F}$ & 0.2 & 0.2 & 0.2 & 0.2 & 0.2 & 0.2 & 0.2 & 0.2 & 0.2 \\
\hline $\mathrm{BZ}$ & \multicolumn{10}{|c|}{} \\
\hline 1 & 10.789 & 11.174 & 11.501 & 10.894 & 11.253 & 11.562 & 11.092 & 11.407 & 11.681 \\
\hline 2 & 11.068 & 11.533 & 11.934 & 11.183 & 11.620 & 12.000 & 11.402 & 11.788 & 12.128 \\
\hline 3 & 11.351 & 11.898 & 12.374 & 11.477 & 11.993 & 12.445 & 11.717 & 12.175 & 12.583 \\
\hline 4 & 10.700 & 11.236 & 11.705 & 10.816 & 11.322 & 11.768 & 11.037 & 11.488 & 11.891 \\
\hline 5 & 11.059 & 11.735 & 12.337 & 11.191 & 11.832 & 12.407 & 11.443 & 12.020 & 12.544 \\
\hline 6 & 12.253 & 13.327 & 14.329 & 12.434 & 13.461 & 14.424 & 12.781 & 13.720 & 14.609 \\
\hline 7 & 12.501 & 13.687 & 14.809 & 12.694 & 13.831 & 14.911 & 13.066 & 14.109 & 15.109 \\
\hline 8 & 12.590 & 13.896 & 15.152 & 12.793 & 14.048 & 15.258 & 13.186 & 14.342 & 15.465 \\
\hline 9 & 13.118 & 14.654 & 16.164 & 13.347 & 14.826 & 16.285 & 13.791 & 15.161 & 16.522 \\
\hline 10 & 14.047 & 16.073 & 18.166 & 14.327 & 16.289 & 18.319 & 14.875 & 16.712 & 18.619 \\
\hline
\end{tabular}

Table 18 Required kilo lumens in to room achieve a cylindrical illuminance of 150 lux for room index 2

\begin{tabular}{|c|c|c|c|c|c|c|c|c|c|}
\hline \multicolumn{10}{|c|}{ Reflectances } \\
\hline C & 0.8 & 0.8 & 0.8 & 0.7 & 0.7 & 0.7 & 0.5 & 0.5 & 0.5 \\
W & 0.7 & 0.5 & 0.3 & 0.7 & 0.5 & 0.3 & 0.7 & 0.5 & 0.3 \\
F & 0.2 & 0.2 & 0.2 & 0.2 & 0.2 & 0.2 & 0.2 & 0.2 & 0.2 \\
\hline BZ & \multicolumn{10}{|c|}{} \\
\hline 1 & 41.073 & 42.097 & 43.038 & 41.665 & 42.582 & 43.427 & 42.809 & 43.524 & 44.185 \\
\hline 2 & 40.372 & 41.440 & 42.424 & 40.953 & 41.914 & 42.802 & 42.076 & 42.834 & 43.538 \\
\hline 3 & 46.577 & 48.124 & 49.568 & 47.366 & 48.770 & 50.083 & 48.902 & 50.033 & 51.092 \\
\hline 4 & 48.659 & 50.456 & 52.146 & 49.533 & 51.173 & 52.717 & 51.239 & 52.576 & 53.835 \\
\hline 5 & 48.403 & 50.410 & 52.313 & 49.294 & 51.137 & 52.886 & 51.035 & 52.561 & 54.009 \\
\hline 6 & 51.071 & 53.986 & 56.830 & 52.140 & 54.856 & 57.504 & 54.244 & 56.567 & 58.827 \\
\hline 7 & 51.326 & 54.427 & 57.473 & 52.422 & 55.320 & 58.161 & 54.584 & 57.076 & 59.513 \\
\hline 8 & 50.578 & 53.817 & 57.020 & 51.668 & 54.702 & 57.697 & 53.818 & 56.443 & 59.024 \\
\hline 9 & 50.571 & 54.013 & 57.443 & 51.683 & 54.915 & 58.129 & 53.879 & 56.690 & 59.474 \\
\hline 10 & 52.808 & 56.954 & 61.166 & 54.063 & 57.977 & 61.942 & 56.553 & 59.999 & 63.468 \\
\hline
\end{tabular}

Table 19 Required kilo lumens in room to achieve a cylindrical illuminance of 150 lux for room index 4

It was also found that when the cylindrical illuminance target was achieved that in all cases the ceiling illuminance target was achieved and in the majority of cases the wall illuminance target was achieved. However it was found that the total flux needed to 
achieve the MRSE target was greater in all cases. Table 20 and Table 21 give the required flux in the room to achieve the MRSE target.

\begin{tabular}{|c|c|c|c|c|c|c|c|c|c|}
\hline \multicolumn{10}{|c|}{ Reflectances } \\
\hline C & 0.8 & 0.8 & 0.8 & 0.7 & 0.7 & 0.7 & 0.5 & 0.5 & 0.5 \\
W & 0.7 & 0.5 & 0.3 & 0.7 & 0.5 & 0.3 & 0.7 & 0.5 & 0.3 \\
F & 0.2 & 0.2 & 0.2 & 0.2 & 0.2 & 0.2 & 0.2 & 0.2 & 0.2 \\
\hline BZ & \multicolumn{10}{|c|}{} \\
\hline 1 & 46.591 & 58.428 & 73.304 & 49.784 & 62.047 & 77.489 & 56.969 & 70.223 & 86.997 \\
\hline 2 & 43.960 & 56.251 & 72.427 & 46.925 & 59.682 & 76.511 & 53.578 & 67.413 & 85.766 \\
\hline 3 & 41.888 & 54.474 & 71.677 & 44.678 & 57.755 & 75.674 & 50.924 & 65.130 & 84.717 \\
\hline 4 & 39.963 & 52.770 & 70.926 & 42.593 & 55.909 & 74.838 & 48.469 & 62.951 & 83.671 \\
\hline 5 & 36.862 & 49.913 & 69.591 & 39.242 & 52.820 & 73.354 & 44.539 & 59.319 & 81.821 \\
\hline 6 & 32.287 & 45.421 & 67.279 & 34.312 & 47.978 & 70.791 & 38.796 & 53.665 & 78.646 \\
\hline 7 & 31.272 & 44.376 & 66.699 & 33.220 & 46.854 & 70.149 & 37.529 & 52.359 & 77.856 \\
\hline 8 & 29.863 & 42.896 & 65.848 & 31.707 & 45.264 & 69.209 & 35.778 & 50.515 & 76.700 \\
\hline 9 & 28.618 & 41.557 & 65.047 & 30.370 & 43.827 & 68.324 & 34.234 & 48.854 & 75.615 \\
\hline 10 & 26.506 & 39.219 & 63.568 & 28.107 & 41.322 & 66.695 & 31.628 & 45.966 & 73.626 \\
\hline
\end{tabular}

Table 20 Required kilo lumens in to room achieve a MRSE of 100 lux for room index 2

\begin{tabular}{|c|c|c|c|c|c|c|c|c|c|}
\hline \multicolumn{10}{|c|}{ Reflectances } \\
\hline C & 0.8 & 0.8 & 0.8 & 0.7 & 0.7 & 0.7 & 0.5 & 0.5 & 0.5 \\
W & 0.7 & 0.5 & 0.3 & 0.7 & 0.5 & 0.3 & 0.7 & 0.5 & 0.3 \\
F & 0.2 & 0.2 & 0.2 & 0.2 & 0.2 & 0.2 & 0.2 & 0.2 & 0.2 \\
\hline BZ & \multicolumn{10}{|c|}{} \\
\hline 1 & 184.48 & 209.41 & 237.57 & 198.36 & 224.38 & 253.86 & 230.50 & 259.25 & 291.88 \\
\hline 2 & 177.91 & 204.81 & 236.12 & 191.11 & 219.29 & 252.19 & 221.62 & 252.93 & 289.62 \\
\hline 3 & 171.07 & 199.88 & 234.52 & 183.60 & 213.85 & 250.34 & 212.43 & 246.20 & 287.12 \\
\hline 4 & 165.88 & 196.04 & 233.24 & 177.90 & 209.62 & 248.86 & 205.50 & 240.98 & 285.12 \\
\hline 5 & 155.73 & 188.27 & 230.52 & 166.78 & 201.06 & 245.72 & 192.03 & 230.48 & 280.92 \\
\hline 6 & 134.43 & 170.75 & 223.77 & 143.54 & 181.84 & 237.97 & 164.17 & 207.11 & 270.58 \\
\hline 7 & 130.38 & 167.21 & 222.29 & 139.14 & 177.97 & 236.28 & 158.93 & 202.45 & 268.34 \\
\hline 8 & 124.74 & 162.18 & 220.11 & 133.02 & 172.48 & 233.79 & 151.68 & 195.84 & 265.04 \\
\hline 9 & 120.16 & 157.98 & 218.22 & 128.05 & 167.90 & 231.63 & 145.81 & 190.35 & 262.21 \\
\hline 10 & 113.18 & 151.40 & 215.12 & 120.50 & 160.74 & 228.10 & 136.91 & 181.81 & 257.57 \\
\hline
\end{tabular}

Table 21 Required kilo lumens in to room achieve a MRSE of 100 lux for room index 4 
The total luminaire flux to achieve the required value of MRSE is very high, however the light distributions do not put any flux directly onto the ceiling, generally the most reflective surface in the room, and so are not efficient at creating MRSE.

The logical solution is to use a light distribution with an upward component, however, the upward flux comes at the expense of downward flux and so the direct component of cylindrical illuminance will reduce and hence it will take a greater total flux in the room to achieve the cylindrical illuminance target. The calculations using upward flux do not require any knowledge of the upward distribution of the light sources as it can be assumed that all upward flux will go directly to the ceiling thus the distribution factor to the ceiling to be set to the upward flux fraction of the light sources and the distribution factors to the walls and ceiling to be scaled so that they add up to the downward light output ratio; direct calculation results are also scaled to reflect the reduced downward flux. Recalculated utilization factors are then used in the calculations of cylindrical illuminance and MRSE.

Figure 2 shows the change in required total flux with changing upward flux fraction ratio for the room that has a room index of 2 with one set of room surface reflection factors. For all flux distributions the flux to achieve the MRSE target decreases as the upward flux fraction increases, whilst the flux need to meet the cylindrical illuminance target increases. If both targets have to be met then the optimum amount of upward flux is found where the two lines cross. 


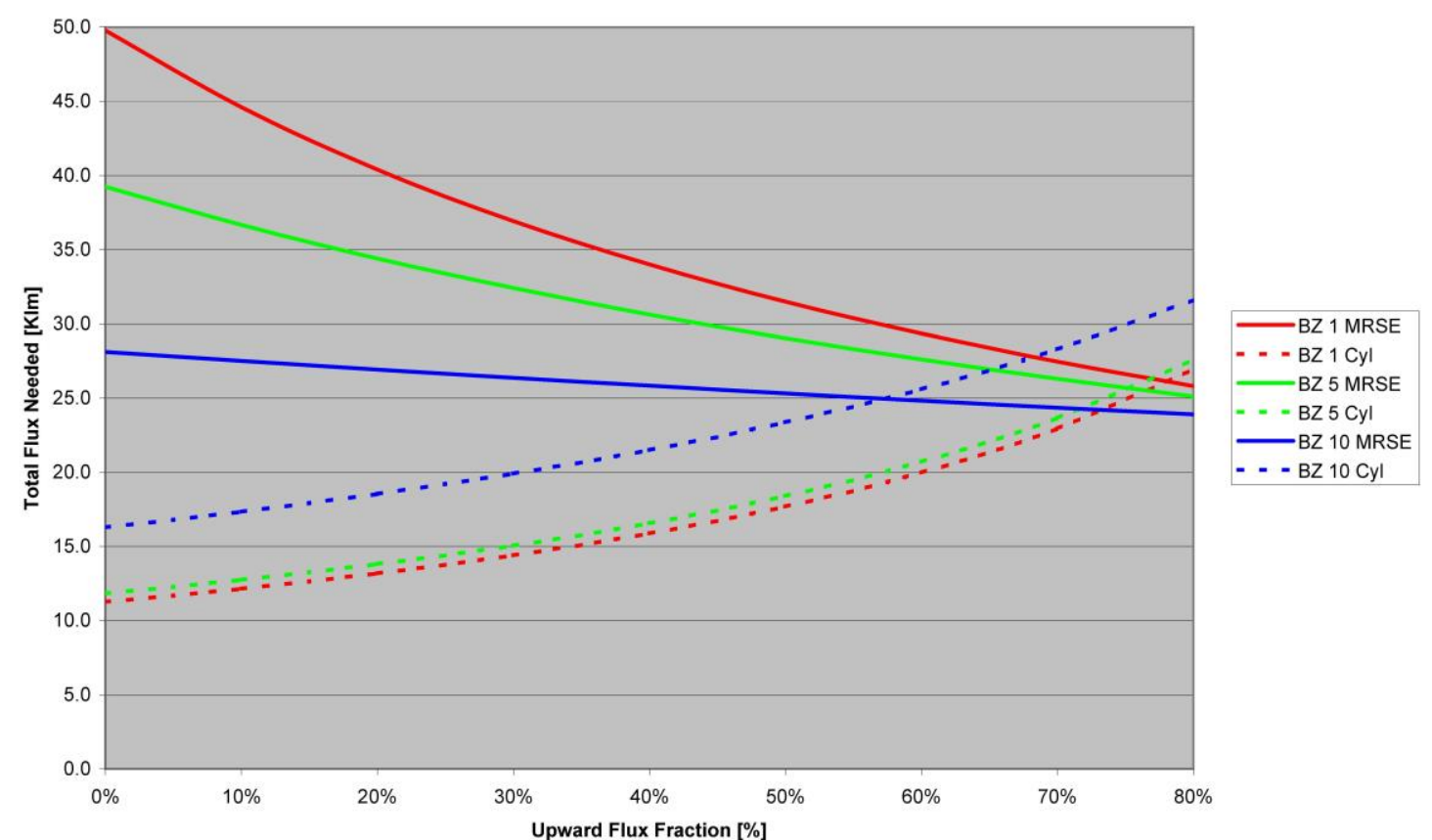

Figure 2 The variation of required total flux with upward flux fraction for a room with room index 2 and reflectances of $0.7,0.5$ and 0.2 for the ceiling, walls and floor respectively

Using this approach it is possible to calculate the optimum amount of upward flux for each flux distribution, room size and combination of room surface reflection factors. Table 22 and Table 23 give the optimum values for the upward flux fraction. 


\begin{tabular}{|c|c|c|c|c|c|c|c|c|c|}
\hline \multicolumn{7}{|c|}{ Reflectances } \\
\hline $\mathrm{C}$ & 0.8 & 0.8 & 0.8 & 0.7 & 0.7 & 0.7 & 0.5 & 0.5 & 0.5 \\
$\mathrm{~W}$ & 0.7 & 0.5 & 0.3 & 0.7 & 0.5 & 0.3 & 0.7 & 0.5 & 0.3 \\
$\mathrm{~F}$ & 0.2 & 0.2 & 0.2 & 0.2 & 0.2 & 0.2 & 0.2 & 0.2 & 0.2 \\
\hline $\mathrm{BZ}$ & \multicolumn{10}{|c|}{} \\
\hline 1 & $79.8 \%$ & $81.7 \%$ & $83.2 \%$ & $82.4 \%$ & $84.0 \%$ & $85.2 \%$ & $87.5 \%$ & $88.5 \%$ & $89.3 \%$ \\
\hline 2 & $79.0 \%$ & $80.9 \%$ & $82.5 \%$ & $81.7 \%$ & $83.3 \%$ & $84.6 \%$ & $86.9 \%$ & $88.0 \%$ & $88.9 \%$ \\
\hline 3 & $78.1 \%$ & $80.2 \%$ & $81.9 \%$ & $80.9 \%$ & $82.6 \%$ & $84.0 \%$ & $86.4 \%$ & $87.5 \%$ & $88.4 \%$ \\
\hline 4 & $79.2 \%$ & $81.2 \%$ & $82.8 \%$ & $81.8 \%$ & $83.5 \%$ & $84.9 \%$ & $87.1 \%$ & $88.2 \%$ & $89.1 \%$ \\
\hline 5 & $77.9 \%$ & $80.1 \%$ & $81.8 \%$ & $80.7 \%$ & $82.5 \%$ & $84.0 \%$ & $86.2 \%$ & $87.4 \%$ & $88.4 \%$ \\
\hline 6 & $73.8 \%$ & $76.6 \%$ & $78.8 \%$ & $77.0 \%$ & $79.4 \%$ & $81.2 \%$ & $83.5 \%$ & $85.0 \%$ & $86.3 \%$ \\
\hline 7 & $72.8 \%$ & $75.7 \%$ & $78.0 \%$ & $76.1 \%$ & $78.6 \%$ & $80.5 \%$ & $82.8 \%$ & $84.4 \%$ & $85.8 \%$ \\
\hline 8 & $71.9 \%$ & $75.0 \%$ & $77.4 \%$ & $75.3 \%$ & $77.9 \%$ & $80.0 \%$ & $82.2 \%$ & $84.0 \%$ & $85.4 \%$ \\
\hline 9 & $69.7 \%$ & $73.2 \%$ & $75.8 \%$ & $73.3 \%$ & $76.3 \%$ & $78.6 \%$ & $80.7 \%$ & $82.7 \%$ & $84.2 \%$ \\
\hline 10 & $65.1 \%$ & $69.4 \%$ & $72.6 \%$ & $69.1 \%$ & $72.8 \%$ & $75.6 \%$ & $77.5 \%$ & $80.0 \%$ & $81.9 \%$ \\
\hline
\end{tabular}

Table 22 Optimum values of upward flux fraction for room index 2

\begin{tabular}{|c|c|c|c|c|c|c|c|c|c|}
\hline \multicolumn{10}{|c|}{ Reflectances } \\
\hline C & 0.8 & 0.8 & 0.8 & 0.7 & 0.7 & 0.7 & 0.5 & 0.5 & 0.5 \\
W & 0.7 & 0.5 & 0.3 & 0.7 & 0.5 & 0.3 & 0.7 & 0.5 & 0.3 \\
F & 0.2 & 0.2 & 0.2 & 0.2 & 0.2 & 0.2 & 0.2 & 0.2 & 0.2 \\
\hline BZ & \multicolumn{10}{|c|}{} \\
\hline 1 & $76.2 \%$ & $77.7 \%$ & $82.0 \%$ & $79.3 \%$ & $80.5 \%$ & $79.7 \%$ & $85.5 \%$ & $86.2 \%$ & n/a \\
\hline 2 & $76.6 \%$ & $78.0 \%$ & $82.6 \%$ & $79.6 \%$ & $80.8 \%$ & $80.2 \%$ & $85.8 \%$ & $86.5 \%$ & n/a \\
\hline 3 & $72.7 \%$ & $74.4 \%$ & $80.1 \%$ & $76.2 \%$ & $77.6 \%$ & $77.5 \%$ & $83.4 \%$ & $84.1 \%$ & n/a \\
\hline 4 & $71.2 \%$ & $73.0 \%$ & $79.4 \%$ & $74.9 \%$ & $76.3 \%$ & $76.6 \%$ & $82.5 \%$ & $83.2 \%$ & n/a \\
\hline 5 & $71.0 \%$ & $72.8 \%$ & $80.1 \%$ & $74.8 \%$ & $76.2 \%$ & $77.2 \%$ & $82.6 \%$ & $83.2 \%$ & n/a \\
\hline 6 & $68.0 \%$ & $70.1 \%$ & $80.4 \%$ & $72.3 \%$ & $73.9 \%$ & $77.2 \%$ & $81.4 \%$ & $81.8 \%$ & n/a \\
\hline 7 & $67.5 \%$ & $69.7 \%$ & $80.6 \%$ & $71.9 \%$ & $73.5 \%$ & $77.4 \%$ & $81.2 \%$ & $81.6 \%$ & n/a \\
\hline 8 & $67.6 \%$ & $69.8 \%$ & $81.4 \%$ & $72.1 \%$ & $73.7 \%$ & $78.2 \%$ & $81.6 \%$ & $81.8 \%$ & n/a \\
\hline 9 & $67.1 \%$ & $69.4 \%$ & $81.9 \%$ & $71.8 \%$ & $73.4 \%$ & $78.6 \%$ & $81.6 \%$ & $81.7 \%$ & n/a \\
\hline 10 & $64.2 \%$ & $66.9 \%$ & $81.8 \%$ & $69.3 \%$ & $71.2 \%$ & $78.2 \%$ & $80.2 \%$ & $80.3 \%$ & n/a \\
\hline
\end{tabular}

Table 23 Optimum values of upward flux fraction for room index 4

Note: in the room with room index 4 at the lowest set of reflectances the flux required to deliver the cylindrical illuminance is always lower than the fluxed needed for MRSE. 
Using the optimum upward flux fraction it is possible to calculate the minimum total flux needed to achieve the MRSE and cylindrical illuminance targets, Table 24 and Table 25 give the values.

\begin{tabular}{|c|c|c|c|c|c|c|c|c|c|}
\hline \multicolumn{10}{|c|}{ Reflectances } \\
\hline C & 0.8 & 0.8 & 0.8 & 0.7 & 0.7 & 0.7 & 0.5 & 0.5 & 0.5 \\
W & 0.7 & 0.5 & 0.3 & 0.7 & 0.5 & 0.3 & 0.7 & 0.5 & 0.3 \\
F & 0.2 & 0.2 & 0.2 & 0.2 & 0.2 & 0.2 & 0.2 & 0.2 & 0.2 \\
\hline BZ & \multicolumn{10}{|c|}{} \\
\hline 1 & 22.215 & 25.389 & 28.714 & 25.460 & 28.966 & 32.654 & 35.852 & 40.442 & 45.244 \\
\hline 2 & 22.198 & 25.448 & 28.838 & 25.420 & 29.005 & 32.760 & 35.755 & 40.421 & 45.303 \\
\hline 3 & 22.202 & 25.494 & 28.943 & 25.401 & 29.048 & 32.868 & 35.653 & 40.406 & 45.389 \\
\hline 4 & 21.939 & 25.254 & 28.733 & 25.152 & 28.812 & 32.646 & 35.404 & 40.182 & 45.190 \\
\hline 5 & 21.877 & 25.294 & 28.900 & 25.045 & 28.827 & 32.796 & 35.187 & 40.114 & 45.289 \\
\hline 6 & 21.862 & 25.545 & 29.443 & 24.931 & 28.992 & 33.309 & 34.741 & 40.040 & 45.633 \\
\hline 7 & 21.826 & 25.594 & 29.587 & 24.868 & 29.017 & 33.433 & 34.591 & 39.995 & 45.705 \\
\hline 8 & 21.706 & 25.566 & 29.675 & 24.714 & 28.976 & 33.499 & 34.333 & 39.860 & 45.734 \\
\hline 9 & 21.679 & 25.683 & 29.976 & 24.632 & 29.046 & 33.758 & 34.072 & 39.805 & 45.932 \\
\hline 10 & 21.563 & 25.904 & 30.583 & 24.403 & 29.178 & 34.320 & 33.458 & 39.637 & 46.291 \\
\hline
\end{tabular}

Table 24 Optimum values for the total flux [KIm] to meet both MRSE and cylindrical illuminance targets for room index $=2$

\begin{tabular}{|c|c|c|c|c|c|c|c|c|c|}
\hline \multicolumn{10}{|c|}{ Reflectances } \\
\hline $\mathrm{C}$ & 0.8 & 0.8 & 0.8 & 0.7 & 0.7 & 0.7 & 0.5 & 0.5 & 0.5 \\
$\mathrm{~W}$ & 0.7 & 0.5 & 0.3 & 0.7 & 0.5 & 0.3 & 0.7 & 0.5 & 0.3 \\
$\mathrm{~F}$ & 0.2 & 0.2 & 0.2 & 0.2 & 0.2 & 0.2 & 0.2 & 0.2 & 0.2 \\
\hline $\mathrm{BZ}$ & \multicolumn{10}{|c|}{} \\
\hline 1 & 85.62 & 92.04 & 96.47 & 97.46 & 104.61 & 113.36 & 135.54 & 144.89 & 151.85 \\
\hline 2 & 85.04 & 91.64 & 96.01 & 96.91 & 104.18 & 112.90 & 134.89 & 144.39 & 151.79 \\
\hline 3 & 86.89 & 93.77 & 97.72 & 98.49 & 106.10 & 114.95 & 135.78 & 145.77 & 151.72 \\
\hline 4 & 87.38 & 94.48 & 98.17 & 98.85 & 106.75 & 115.61 & 135.79 & 146.09 & 151.66 \\
\hline 5 & 86.63 & 94.10 & 97.57 & 97.99 & 106.27 & 114.97 & 134.64 & 145.41 & 151.54 \\
\hline 6 & 85.77 & 94.40 & 97.12 & 96.77 & 106.23 & 114.57 & 132.28 & 144.43 & 151.22 \\
\hline 7 & 85.44 & 94.31 & 96.93 & 96.36 & 106.11 & 114.33 & 131.68 & 144.11 & 151.15 \\
\hline 8 & 84.59 & 93.75 & 96.31 & 95.43 & 105.46 & 113.59 & 130.61 & 143.37 & 151.05 \\
\hline 9 & 84.07 & 93.54 & 95.91 & 94.79 & 105.16 & 113.18 & 129.78 & 142.87 & 150.95 \\
\hline 10 & 81.50 & 94.06 & 95.87 & 94.30 & 105.50 & 113.29 & 128.54 & 142.48 & 150.79 \\
\hline
\end{tabular}

Table 25 Optimum values for the total flux [Klm] to meet both MRSE and cylindrical illuminance targets for room index $=4$ 
Table 26 and Table 27 give the relative increase in flux required if a MRSE value of 100 lux was required, compared to the current situation where the flux requirement based on supplying a cylindrical illuminance of 150 lux.

\begin{tabular}{|c|r|r|r|r|r|r|r|r|l|}
\hline \multicolumn{10}{|c|}{ Reflectances } \\
\hline C & 0.8 & 0.8 & 0.8 & 0.7 & 0.7 & 0.7 & 0.5 & 0.5 & 0.5 \\
F & 0.7 & 0.5 & 0.3 & 0.7 & 0.5 & 0.3 & 0.7 & 0.5 & 0.3 \\
\hline BZ & 0.2 & 0.2 & 0.2 & 0.2 & 0.2 & 0.2 & 0.2 & 0.2 & 0.2 \\
\hline 1 & $106 \%$ & $127 \%$ & $150 \%$ & $134 \%$ & $157 \%$ & $182 \%$ & $223 \%$ & $255 \%$ & $287 \%$ \\
\hline 2 & $101 \%$ & $121 \%$ & $142 \%$ & $127 \%$ & $150 \%$ & $173 \%$ & $214 \%$ & $243 \%$ & $274 \%$ \\
\hline 3 & $96 \%$ & $114 \%$ & $134 \%$ & $121 \%$ & $142 \%$ & $164 \%$ & $204 \%$ & $232 \%$ & $261 \%$ \\
\hline 4 & $105 \%$ & $125 \%$ & $145 \%$ & $133 \%$ & $154 \%$ & $177 \%$ & $221 \%$ & $250 \%$ & $280 \%$ \\
\hline 5 & $98 \%$ & $116 \%$ & $134 \%$ & $124 \%$ & $144 \%$ & $164 \%$ & $207 \%$ & $234 \%$ & $261 \%$ \\
\hline 6 & $78 \%$ & $92 \%$ & $105 \%$ & $101 \%$ & $115 \%$ & $131 \%$ & $172 \%$ & $192 \%$ & $212 \%$ \\
\hline 7 & $75 \%$ & $87 \%$ & $100 \%$ & $96 \%$ & $110 \%$ & $124 \%$ & $165 \%$ & $183 \%$ & $203 \%$ \\
\hline 8 & $72 \%$ & $84 \%$ & $96 \%$ & $93 \%$ & $106 \%$ & $120 \%$ & $160 \%$ & $178 \%$ & $196 \%$ \\
\hline 9 & $65 \%$ & $75 \%$ & $85 \%$ & $85 \%$ & $96 \%$ & $107 \%$ & $147 \%$ & $163 \%$ & $178 \%$ \\
\hline 10 & $54 \%$ & $61 \%$ & $68 \%$ & $70 \%$ & $79 \%$ & $87 \%$ & $125 \%$ & $137 \%$ & $149 \%$ \\
\hline
\end{tabular}

Table 26 Relative increase in total flux needed to meet the additional MRSE target for room index $=2$

\begin{tabular}{|c|r|r|r|r|r|r|r|r|r|}
\hline \multicolumn{10}{|c|}{ Reflectances } \\
\hline C & 0.8 & 0.8 & 0.8 & 0.7 & 0.7 & 0.7 & 0.5 & 0.5 & 0.5 \\
F & 0.7 & 0.5 & 0.3 & 0.7 & 0.5 & 0.3 & 0.7 & 0.5 & 0.3 \\
\hline BZ & 0.2 & 0.2 & 0.2 & 0.2 & 0.2 & 0.2 & 0.2 & 0.2 & 0.2 \\
\hline 1 & $108 \%$ & $119 \%$ & $124 \%$ & $134 \%$ & $146 \%$ & $161 \%$ & $217 \%$ & $233 \%$ & $244 \%$ \\
\hline 2 & $111 \%$ & $121 \%$ & $126 \%$ & $137 \%$ & $149 \%$ & $164 \%$ & $221 \%$ & $237 \%$ & $249 \%$ \\
\hline 3 & $87 \%$ & $95 \%$ & $97 \%$ & $108 \%$ & $118 \%$ & $130 \%$ & $178 \%$ & $191 \%$ & $197 \%$ \\
\hline 4 & $80 \%$ & $87 \%$ & $88 \%$ & $100 \%$ & $109 \%$ & $119 \%$ & $165 \%$ & $178 \%$ & $182 \%$ \\
\hline 5 & $79 \%$ & $87 \%$ & $87 \%$ & $99 \%$ & $108 \%$ & $117 \%$ & $164 \%$ & $177 \%$ & $181 \%$ \\
\hline 6 & $68 \%$ & $75 \%$ & $71 \%$ & $86 \%$ & $94 \%$ & $99 \%$ & $144 \%$ & $155 \%$ & $157 \%$ \\
\hline 7 & $66 \%$ & $73 \%$ & $69 \%$ & $84 \%$ & $92 \%$ & $97 \%$ & $141 \%$ & $152 \%$ & $154 \%$ \\
\hline 8 & $67 \%$ & $74 \%$ & $69 \%$ & $85 \%$ & $93 \%$ & $97 \%$ & $143 \%$ & $154 \%$ & $156 \%$ \\
\hline 9 & $66 \%$ & $73 \%$ & $67 \%$ & $83 \%$ & $91 \%$ & $95 \%$ & $141 \%$ & $152 \%$ & $154 \%$ \\
\hline 10 & $54 \%$ & $65 \%$ & $57 \%$ & $74 \%$ & $82 \%$ & $83 \%$ & $127 \%$ & $137 \%$ & $138 \%$ \\
\hline
\end{tabular}

Table 27 Relative increase in total flux needed to meet the additional MRSE target for room index $=4$ 


\section{Conclusion}

This paper has reviewed the current and proposed metrics for illumination in a room where no defined visual task is present for a set light distributions.

Of the current requirements in EN $12464-1^{1}$ it clear that the cylindrical illuminance is the most onerous. The proposed metric of MRSE was studied and it was found that with no upward light that a significant increase in the total amount of flux was needed to achieve a value of 100 lux as compared to the flux required to give a cylindrical illuminance of 150 lux.

It was found that by adding an upward component to the flux distribution the MRSE value could be achieved with a lower total flux, however, distributions with upward flux increased the amount of flux needed to meet the cylindrical illuminance target. It is thus necessary to find an optimum upward flux ratio to calculate minimum total flux to meet both cylindrical illuminance and MRSE targets. However in all cases considered the addition of the MRSE target increased the total flux needed.

Adding the requirement for MRSE to current practice would encourage the use of wide distribution luminaires with at least $60 \%$ upward flux and high reflection factors for room surfaces.

However, the adoption of a MRSE target of 100 lux will require a significant increase in flux used. This will in turn lead to more energy being used to provide it. At a time when there is significant restraint on energy used it is going to be difficult to persuade the standards makers to adopt a MRSE target with a value so high. 
${ }^{1}$ BS EN 12464-1: 2011: Light and lighting - Lighting of work places - Part 1: Indoor work places, London: BSI.

${ }^{2}$ Cuttle C. Towards the third stage of the lighting profession Lighting Res. Technol.2010; 42:73-93

${ }^{3}$ BS EN 13032-2:2004 Light and lighting - Measurement and presentation of photometric data of lamps and luminaires - Part 2: Presentation of data for indoor and outdoor workplaces.

${ }^{4}$ Raynham P. J. and Bean A. R. Calculation of transfer factors in the European utilization factor method Lighting Res. Technol. 2006; vol. 38, 4: pp. 341-357.

${ }^{5}$ The SLL Code for Lighting, 2012, CIBSE London, ISBN 9781906846213 